\title{
Protocol Deviation End Time
}

National Cancer Institute

\section{Source}

National Cancer Institute. Protocol Deviation End Time. NCI Thesaurus. Code C83048.

The stop time of the protocol deviation. 